\title{
TRATAMENTO ENDODÔNTICO DE CANAIS CALCIFICADOS COM AUXÍLIO DA ENDODONTIA GUIADA
}

\author{
Milena Vieira ${ }^{1}$ \\ Pamela Freitas Aguiar ${ }^{2}$
}

\begin{abstract}
RESUMO: A endodontia é uma área da Odontologia que tem como objetivo tratar o dente internamente, recuperando a saúde dentária. Em suma, é um tratamento complexo, que abrange várias complicações. Dentro da endodontia discute-se muito sobre canais calcificados sendo eles de origem patológica ou não, podendo envolver desafios em sua execução e conclusão. $O$ tratamento do sistema de canal radicular calcificado através da endodontia guiada vem sendo ótimo aliado para a realização destes casos. É realizado com associação entre tomografia computadorizada, escaneamento digital e prototipagem através de impressão ${ }_{3} \mathrm{D}$. A Endodontia guiada se apresenta como uma alternativa eficaz e necessária para solucionar casos mais complexos, como tratamento de canais radiculares calcificados, microcirurgias endodônticas, ainda que haja algumas limitações nesta técnica, se tornam irrelevantes diante dos benefícios. O objetivo desse trabalho é realizar uma revisão de literatura, entre os anos de 1967 á 2020, justifica-se através do estudo o tratamento de canais calcificados utilizando a odontologia guiada como forma de tratamento, tornando o procedimento mais simples, eficaz e diminuindo o tempo de trabalho.
\end{abstract}

Palavras-chaves: Endodontia. Canais calcificados. Endodontia guiada. Endoguide.

ABSTRACT: Endodontics is an area of Dentistry that aims to treat the tooth internally, restoring dental health. In short, it is a complex treatment that covers several complications. Within endodontics, much is discussed about calcified canals, whether they are of pathological origin or not, which may involve challenges in their execution and conclusion. The treatment of the calcified root canal system through guided endodontics has been an excellent ally for the realization of these cases. It is performed with association between computed tomography, digital scanning and prototyping through $3 \mathrm{D}$ printing. Guided endodontics is an effective and necessary alternative to solve more complex cases, such as the treatment of calcified root canals, endodontic microsurgeries, although there are some limitations in this technique, they become irrelevant given the benefits. The objective of this work will be to carry out a literature review, between the years 1967 to 2020 , it is justified through the study the treatment of calcified canals using

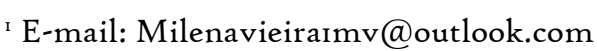


guided dentistry as a form of treatment, making the procedure simpler, more effective and reducing time of work.

Keywords: Dentistry. Endodontics. Calcified channels. Guided endodontics. Endoguide

\section{INTRODUÇÃO}

$\mathrm{Na}$ anatomia dentária é indispensável abordar sobre o complexo dentino pulpar, sendo esse uma região suscetível a sofrer danos, capazes de gerar obliteração dos canais radiculares, este fenômeno é causado por diversos fatores, como cáries, envelhecimentos, traumas, dentre outros que podem atingir a estrutura dentária. Fisiologicamente, os odontoblastos passam a depositar uma quantidade de dentina secundária (em casos de idade, onde é depositada lentamente com o tempo) ou terciária (caso se trate de alguma lesão ou trauma), sendo esta última mais desorganizada e depositada mais rapidamente. As massas de tecido calcificado no nível da câmara pulpar e canais radiculares do dente são denominados de Calcificação Pulpar (CP). (BUCHGREITS J. 2016), (NEVILLE B. ALLEN C. 2004), (SATHEESHKUMAR

P. 2013).

A CP pode estar presente no nível da câmara pulpar e/ou-canal radicular. ${ }^{3}$ podendo até obliterar o canal radicular completamente e esta não pressupõe obrigatoriamente alterações na cor da coroa do dente, no periápice do dente e na sensibilidade do dente ${ }^{4}$. Podem ocorrer em dentes saudáveis, doentes, irrompidos ou impactados em todas as faixas etárias 6. (SATHEESHKUMAR P. 2013), (MCCABE P. DUMMER P. 2012), (TASSOKER M., MAGAT G. SENNER S. 2018).

As calcificações podem ser localizadas mais regularmente na porção coronária do que na porção radicular do órgão pulpar. As ocorrências relatadas divergem de $8 \%$ a 90\%, mas diferentes pesquisadores registraram a prevalência em quase $20 \%$ de dentes registrados individualmente por meio de radiografias. Nas calcificações pulpares as prevalências, parecem aumentar com a idade dos indivíduos devido a inúmera quantidade de impactos físicos e químicos, aos quais os dentes são constantemente expostos ao longo da vida dos indivíduos, até chegar à senilidade. (RAVANSHAD S. 2015). 
Destaca-se entre as formas mais comuns de deposição de tecido mineralizado a produção localizada da dentina reacional, em consequência à agressão bacteriana provocada por cárie. As calcificações também estão ligadas a causas fisiológicas oriundas da senilidade do tecido pulpar onde pesquisas mostram que o surgimento de áreas mineralizadas em polpa madura, está relacionado ao processo de envelhecimento deste órgão Outras possíveis causas são distúrbios circulatórios da polpa, causas idiopáticas, movimentação no tratamento ortodôntico, e predisposição genética. (MORELI A. MOREIRA L. RABELLO D. 2010), (RAVANSHAD S. 2015), (LUUKKO K. 20II).

Devido à dificuldade de encontro dos canais, nessas condições pode acontecer uma grande margem de erros, dentre esses está o grande risco de perfuração, podem acontecer também desvios que favorecem o insucesso do tratamento, outro problema bastante recorrente é a fratura de limas, pois muitas vezes são desempenhadas forças superiores às suportadas pelos instrumentos endodônticos ou estes são empregados de forma incorreta, e o comum caso de não conseguir acesso aos canais devido a calcificação, assim acabam comprometendo a estrutura dentária íntegra. Logo a American Association of Endodontists classifica o tratamento de dentes com calcificação dos canais pulpares como de alto nível de dificuldade. Conforme as dificuldades, foram desenvolvidos recursos clínicos como, radiografias, microscópio, ultrassom, e tomografia, com o intuito de trazer melhoras a esse tratamento. (DORANALA S. 2020), (AMERICAN A. S. E. 20i6), (BUCHGREITS J. 2016).

Além desses equipamentos e exames foi introduzido um conceito chamado "Endodontia Guiada". Este tratamento permite reduzir o tempo de cadeira e o risco de dano iatrogênico a estrutura dentária. Outro aspecto valioso é que a curva de aprendizado para realizar essa técnica é pequena, permitindo que profissionais menos experientes a realizem. Na realização do planejamento são necessários outros recursos tecnológicos, trabalhando em conjunto para que o modelo de orientação final tenha eficácia, este chamado Endoguide. (KRASTL G. 2016), (ALI A. 2019), (CASADEI B. 2020).

Entendendo a importância dessa nova tecnologia no contexto atual da terapia endodôntica e seu crescente emprego este estudo tem como objetivo, realizar uma revisão 
de literatura sobre a Endodontia Guiada no tratamento de canais calcificados, abordando a técnica, suas aplicações, vantagens e desvantagens, em busca de um melhor prognóstico em longo prazo, melhorias na execução desse tratamento, no tempo de trabalho. É imprescindível o conhecimento sobre essa inovação tecnológica que vem tomando devida proporção sobre a endodontia, e na execução do tratamento dos canais calcificados. Esse estudo torna-se essencial devido um grande fracasso no tratamento endodôntico de canais calcificados, nesse estudo abordaremos as vantagens do uso de guias endodônticos, especialmente para profissionais voltados a aérea da endodontia e na área Bucomaxilo.

Para realização desta revisão de literatura foram utilizados como base científica, mais de 19 artigos científicos a respeito do tema escolhido, encontrados em plataformas online como Scientific Eletronic Library On-line (Scielo) e Google acadêmicos, publicados no período entre 2005 e 2019 .

Como critério de inclusão para a escolha dos materiais utilizados, serão realizadas pesquisas online empregando palavras chaves como: Endodontia, Canais, Calcificados, Endoguide, Camara pulpar, tratamentos, Tecnologia, entre outras.

\section{REVISÃO DE LITERATURA}

\section{I. Complexo Dentino Pulpar}

O complexo pulpo-dentinário é formado, assim como o nome indica, pela polpa e dentina que são tecidos que partilham a mesma origem embrionária (tecido mesenquimatoso) e que, desde à formação do dente, apresentam uma relação íntima entre si. Contudo, apesar de intimamente ligados, contém características diferentes (LOPES H. 2015)

Este complexo encontra-se protegido dos agentes agressores presentes no meio oral pelo esmalte a nível coronal e pelo cimento a nível radicular. Quando estas estruturas são afetadas, o complexo fica exposto a agentes agressores. Desta maneira, como meio de resposta a estes agentes, ocorrem modificações na dentina que irão ser reproduzir a nível pulpar e as modificações pulpares (dor, inflamação, etc) irão causar modificações dentinárias. (COHEN S. HARGREAVES K. 2011), (LOPES H. 2015) 
A dentina é um tecido mineralizado que reveste a polpa e que cria paredes na câmara pulpar, com quem estabelece ligação através dos seus túbulos dentinários, e canal radicular. A polpa é composta por odontoblastos que são as células responsáveis pela dentinogenese, tanto durante o desenvolvimento do dente assim como durante a sua maturação. Desta forma, o odontoblasto é a célula mais característica do complexo pulpodentinário. Durante a dentinogénese os odontoblastos formam os túbulos dentinários e a sua aparição dentro dos túbulos faz da dentina um tecido vivo receptivo. (COHEN S. HARGREAVES K. 20II)

Quando está sobre um fator agressor a polpa contestara por meio de reações de defesa, que poderão ser inflamatórias ou degenerativas e estarão presentes de acordo do tipo, da constância e da intensidade do agente agressor. Se essas reações não forem suspensas por intermédio da remoção da causa, poderá causar o envelhecimento pulpar, calcificação do canal radicular, palpites ou mesmo necrose pulpar. (LOPES H. 2015)

O complexo pulpo-dentinário expõe mecanismos de defesa tais como deposição de dentina terciária, deposição de dentina intratubular e reações inflamatórias cujos objetivos são manter a vitalidade do tecido e dos odontoblastos contra agressões de origem: biológica, térmica, química ou mecânica. No entanto, quando estes mecanismos de defesa fracassam ocorre suprimento neuro vascular do tecido pulpar levando a uma diminuição do tecido pulpar, e por recorrência aumento do tecido mineralizado no interior da cavidade pulpar e canais radiculares. (LOPES H. 2015)

\subsection{Canais Calcificados}

O acúmulo de tecido mineralizado na câmara pulpar ou nos canais radiculares dos dentes dão origem as calcificações pulpares. Elas têm um grande potencial de levar complicações para a prática clínica odontológica, em decorrência da obliteração e modificação na anatomia pulpar. As calcificações são comumente resultado de respostas fisiológicas ao processo de envelhecimento pulpar, mas também podem ter relação com aspectos ambientais, como traumatismos, cáries e hábitos para funcionais. É descoberta através de anamnese, exame clínico e exames complementares para diagnóstico definitivo. 
$\mathrm{Na}$ anamnese pode ser mencionado pelo paciente se ocorreu algum tipo de trauma na região dos dentes com suspeita de calcificação ou se o mesmo faz uso de agentes químicos como o cigarro ou drogas ilícitas. (MEDEIROS B. 2017)

O mecanismo fundamental que leva ao desenvolvimento da calcificação pulpar não é conhecido. Entretanto, a ruptura do feixe neuro-vascular aparenta estimular a rápida construção de tecido mineralizado. A calcificação desperta o seu desenvolvimento na câmara pulpar e progride ao longo das paredes dos canais radiculares com o passar do tempo. As calcificações podem ocorrer em jovens ou idosos e em um ou mais dentes. (MCCABE P. DUMMER P. 2012), (TASSOKER M., MAGAT G. SENNER S. 2018)

Figura I: ilustrando um canal calcificado.

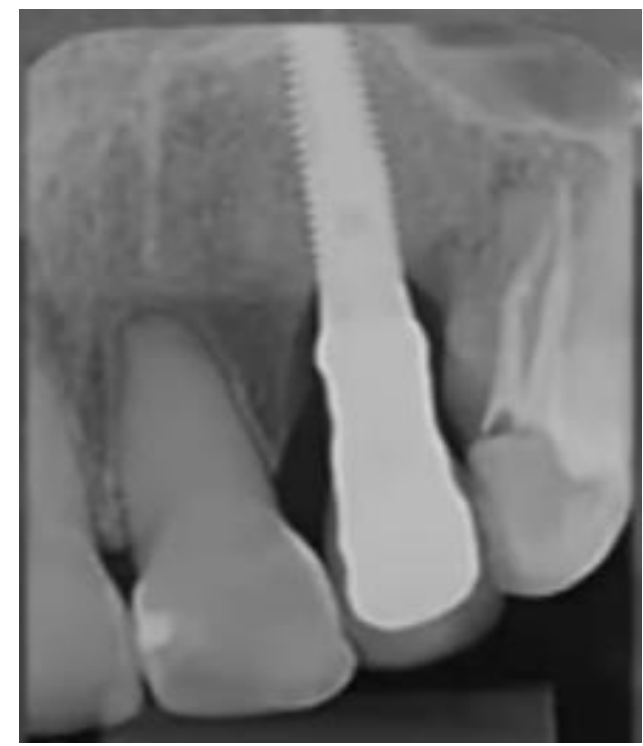

Fonte: Holcomb Gregory et al. (1967)

\subsection{Diagnóstico}

Desenvolver um diagnóstico preciso tem como principal finalidade determinar qual o melhor tratamento a ser realizado. Desse modo, este é o ponto-chave para um parecer clínico. Se for feito um diagnóstico indevido, será também feito um tratamento indevido e inapropriado podendo ter consequências leves a severas. (CORROTTE P. 2005) 
O maior número de dentes com calcificação pulpar é considerado saudáveis e funcionais após realizar o exame clínico, e radiográfico, bem como determinação dos sinais e sintomas. Dentes que sofrem de obliteração pulpar são frequentemente assintomáticos e são diagnosticados certas vezes de forma acidental. (OGINNI A. 2009)

O diagnóstico de dentes que manifestam calcificações pulpares é feito através de: exame clínico, histológico, exames radiográficos, e execução de testes de sensibilidade pulpar. Cada um dos métodos de diagnóstico apresenta-se na tabela 3 dos anexos. . (MCCABE P. DUMMER P. 2012), (TASSOKER M., MAGAT G. SENNER S. 2018)

Diante de dentes calcificados o aparecimento ou falta de sintomas está vinculado ao estado da mineralização do sistema de canais. Normalmente a resposta ao frio e ao quente pode exibir-se normal ou ausente, conforme a fase da calcificação. Normalmente estes dentes não apontam sensibilidade a percussão. (CAMPOS M. 2016).

No exame complementar radiográfico observa-se que não há presença de imagem radiopaca no interior da câmera pulpar e canais radiculares, e sim, imagem única radiolúcida que seria o canal calcificado. Essa calcificação pode estar tanto na porção coronária quanto na porção radicular. (MEDEIROS B. 2017).

Em relação ao diagnóstico por imagem, a confiabilidade da determinação de calcificação pulpar com radiografias interproximais ou periapicais é superior quando confrontada às radiografias panorâmicas. Entretanto, como a radiografia panorâmica produz uma única imagem de ambos os arcos, maxilar e mandibular, ela também pode ser considerada uma ferramenta válida para triagem inicial da presença de calcificações. (MOVAHHENDIAM M. HAGHNNEGAHDAR A. OWII F.2018)

Neste contexto a Tomografia Computadorizada Cone Beam (TCCB) possibilita a formação de imagem tridimensional dos tecidos mineralizados maxilofaciais, com mínima deformação e quantidade de radiação significantemente reduzida em conferência à tomografia computadorizada tradicional. (SCARF W. FARMAN A. SOKOVIC P. 2006)

Em estudo, afirmaram que a Tomografia Computadorizada de Feixe Cônico (TCFC) é uma ferramenta de diagnóstico valiosa para avaliar a câmara pulpar e a calcificação da polpa, pois fornece detalhes anatômicos precisos em três dimensões, 
oferecendo a possibilidade de visualizar e medir cada dente individualmente em vistas axiais, sagitais e coronais, sem quaisquer sobreposições. (CAGLAYAN F. DAGISTAN S. KEES M. 2015), (ABELLA F. 2015).

Segue em anexo as imagens captadas pela TCFC em suas dimensões, e os aparelhos utilizados para o tratamento

Figura 2: vista sagital
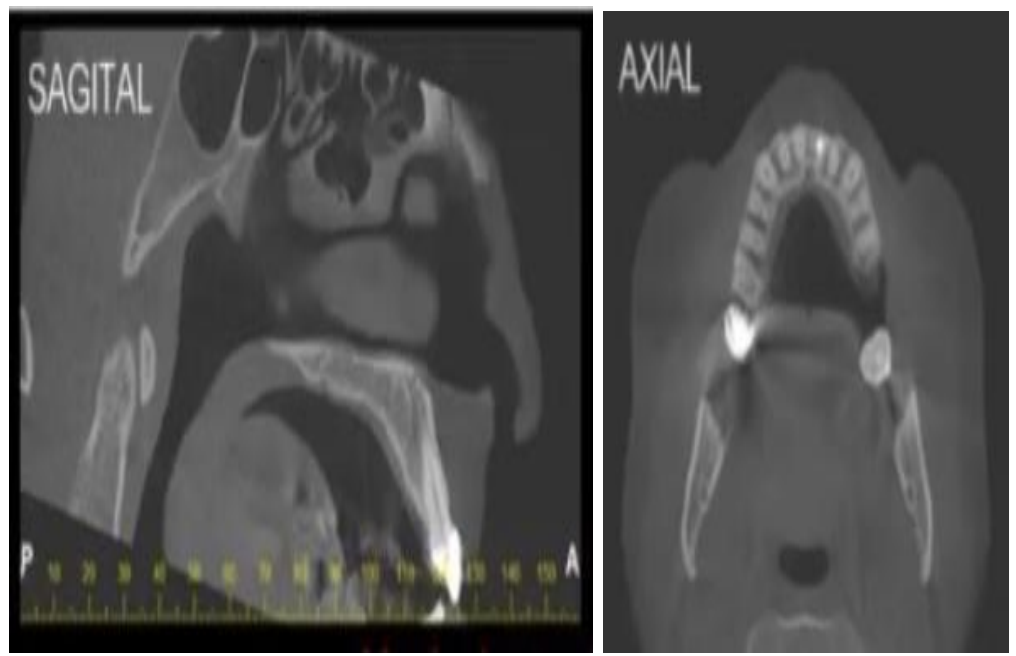

Figura 4: vista coronal

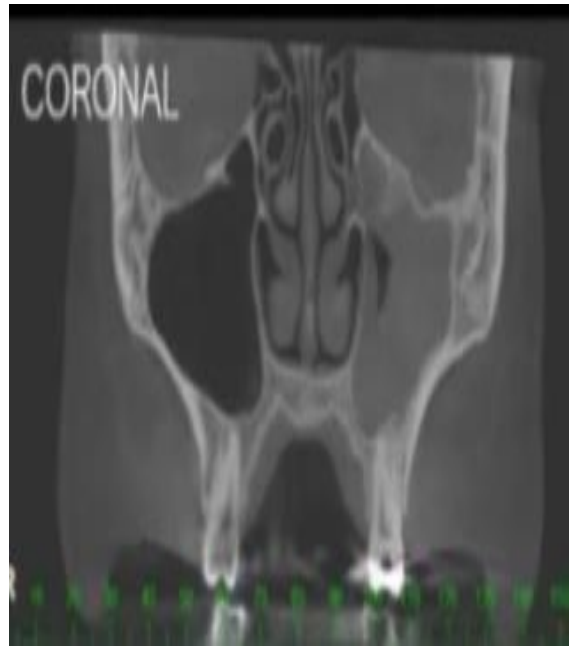

Fonte: Dental scan et al. (2005)

Figura 5: TOMOGRAFIA COMPUTADORIZADA CONE BEAM

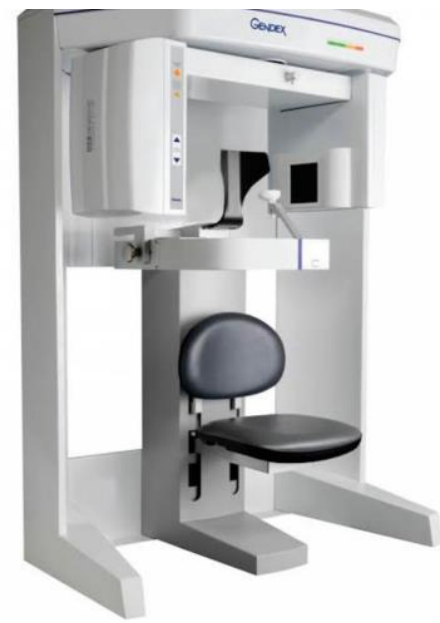

Fonte: Mozzo et. al (1998) 
Figura 6: Scan-intra oral

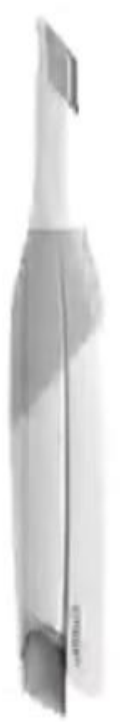

Fonte: Mozzo et al. (1998)

Figura 7: Impressora 3D

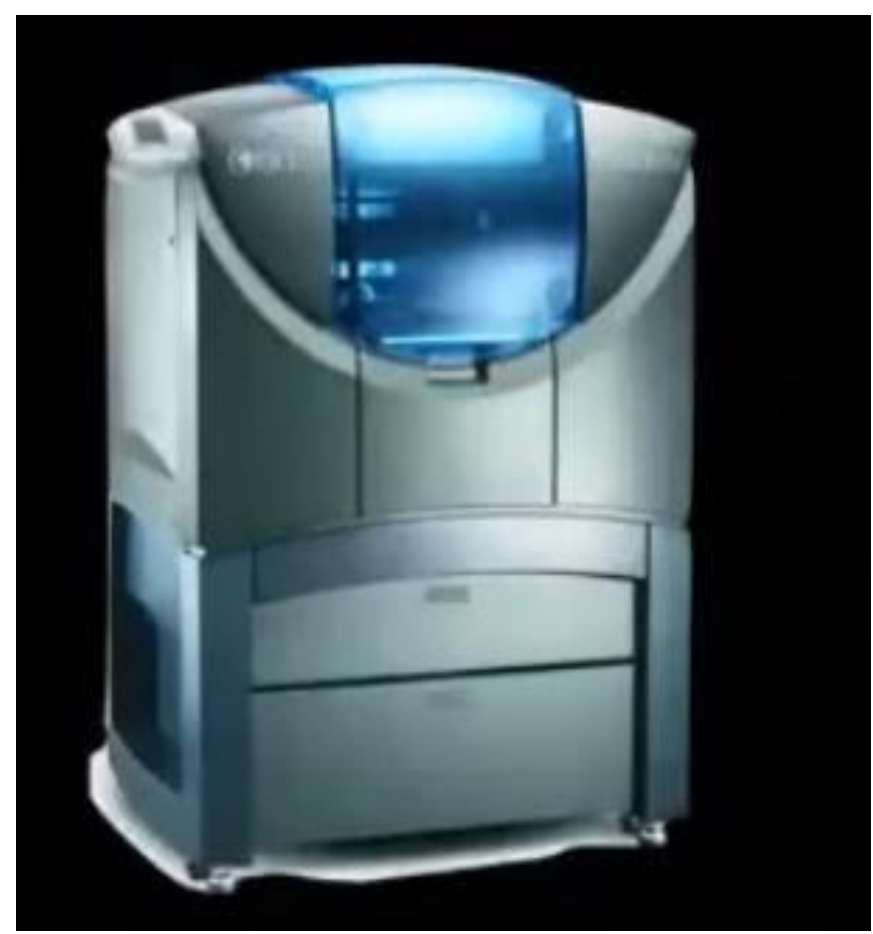

Fonte: Mozzo et al. (1998) 


\section{4. Endodontia Guiada}

Nos últimos anos, com a expansão do uso das TCFC, scan-intra oral e planejamento virtual de implantes usando softwares apropriados, novas técnicas têm sido desenvolvidas para casos clínicos de CP que necessitam de tratamento endodôntico, surgindo assim a técnica

com guias. O tratamento endodôntico guiado, tem sido realizado em diferentes pesquisas, fazendo avaliação, planejamento e execução, seguindo o mesmo padrão.( MENDES L. 2018), (TAVARES W. 2018), (CONNERT T. 2018)

Com base nesses escaneamentos, foram desenvolvidos guias endodônticos para o tratamento planejado por meio de projeto digital e fabricação de prototipagem rápida. Os guias feitos sob medida possibilitaram uma localização e gestão descomplicada e previsível do canal. O método de design digital e prototipagem rápida de guias endodônticos possibilitou a localização confiável e previsível dos canais radiculares dos dentes com sistemas de canais radiculares calcificados. (MENDES L. 2018)

Com o uso da tomografia e impressão $3 \mathrm{D}$, surgiu o conceito de endodontia guiada, validando um método seguro e clinicamente acessivel para localizar canais radiculares e evitar a perfuração do canal em dentes com CP. Através da sobreposição da tomografia com o escaneamento intra oral, que já são apresentadas diversas marcas e modelos, em conjunto com o planejamento virtual em software especializado, obteremos uma precisão para o guia endodôntico. (KRASTL G.2016)

Após o planejamento, o modelo virtual será exportado como um arquivo STL e enviado para a impressora $3 \mathrm{D}$ que imprimirá o guia. I Estereolitografia (SLA) faz a confecção da prototipagem rápida, com o material de polímero de resina líquida (acrílico, epóxi ou vinil) e, em seguida, tenha uma réplica fiel ao modelo virtual denominado protótipo biológico, por meio desse processo, obtivemos um guia de acesso. Em seguida da fixação guiada e acesso ao canal radicular, o tratamento endodôntico segue os métodos convencionais. O Endoguide foi confeccionado conforme a tomografia e plano de varredura intraoral. A endodôntica guiada é uma técnica desenvolvida para preparar a cavidade para acesso ao canal radicular. Fornecido em linha reta e de maneira precisa, 
fazendo uma guia para permitir a entrada da broca especialmente projetada e precisa, para a referida parte de acesso ao canal radicular,

Comumente está localizado no terço apical da raiz. Essa é uma técnica que traz resultados adequados e previsíveis. Para execução dessa técnica, são indispensáveis os dados da tomografia computadorizada de feixe cônico, visto que possibilita uma visualização tridimensional das estruturas anatômicas, além de proporcionar as medidas da borda incisal do dente até o ápice radiográfico (figuras 8 e 9). (ZEHNDER M. 2015).

Figura 8: Superposição da broca virtual Figura 9: Broca virtualmente sobreposta ao no dente para formar o cesso em linha canal radicular no software de planejamento. reta ao terço apical da raiz do canal.

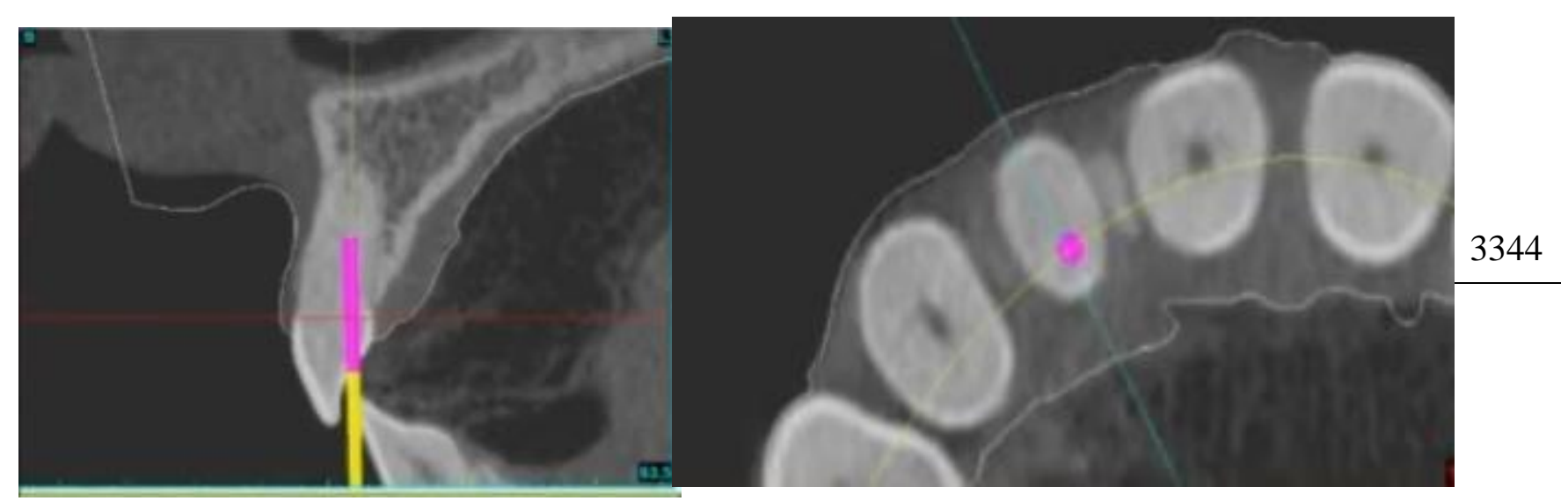

Fonte: Zehnder et al. (2015)

Fonte: Zehnder et al. (2015)

São armazenados como arquivos digitais os dados das imagens extraídos da TCFC. É realizado uma inspeção intra orais com scanners de superfície ${ }_{3} \mathrm{D}$ são executadas na cavidade oral do paciente. Todas essas informações são abrigadas em softwares de planejamento projetados para cirurgia de implante guiada. Entre mais conhecidos e populares, têm-se o coDiagnostiX. Esses softawes produzem uma imagem virtual da broca, que será sobreposta em cada dente, com o objetivo de criar um acesso direto ao terço apical do canal radicular e, posteriormente ela será confeccionada. (CONNERT T. 2017). 
Figura Io: Planejamento virtual: Figura Ir: Planejamento virtual: visualização visualização $3 \mathrm{D}$ de cavidades de acesso $3 \mathrm{D}$ de cavidades de acesso planejadas, planejadas, incluindo brocas e modelo.

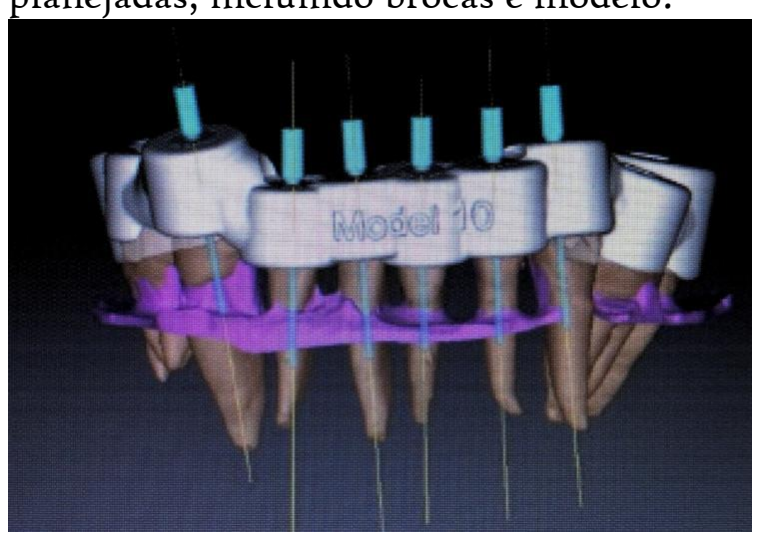
incluindo brocas e modelo.

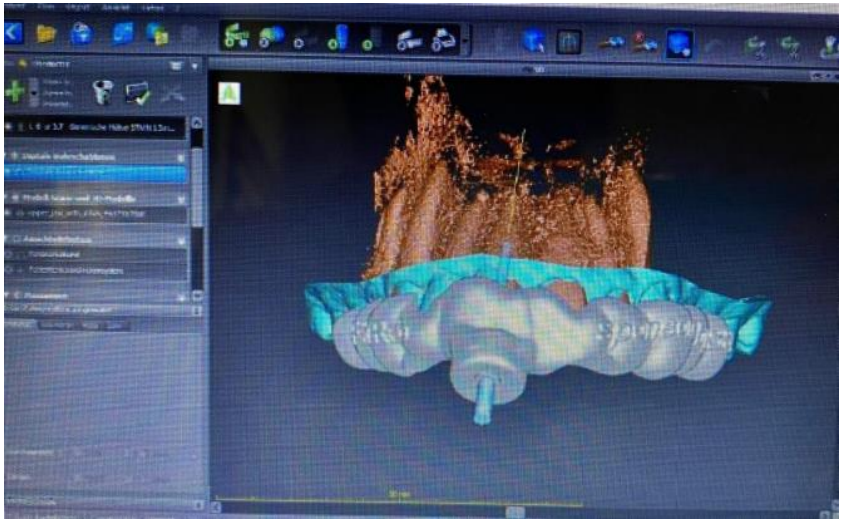

Fonte: CONNERT et al. (2017)

Fonte: CONNERT et al. (2017)

O software cria também um modelo virtual com guias de aceso ao canal radicular, e em seguida o modelo é impresso em uma impressora ${ }_{3} \mathrm{D}$. Uma tecnologia de Controle Numérico Computadorizada (CNC) é usada para criar a manga projetada, que é integrada no modelo impresso para orientar a broca durante a preparação da cavidade. Essas mangas de fixação são desenvolvidas com a finalidade de estabilizar o guia, impedindo que a broca desvie sua trajetória, provocando perfurações. É adaptada no modelo impresso para guiar a broca durante a preparação da cavidade. (MENDES L. 2018)

Figura 12: Prototipagem do guia endodôntico.

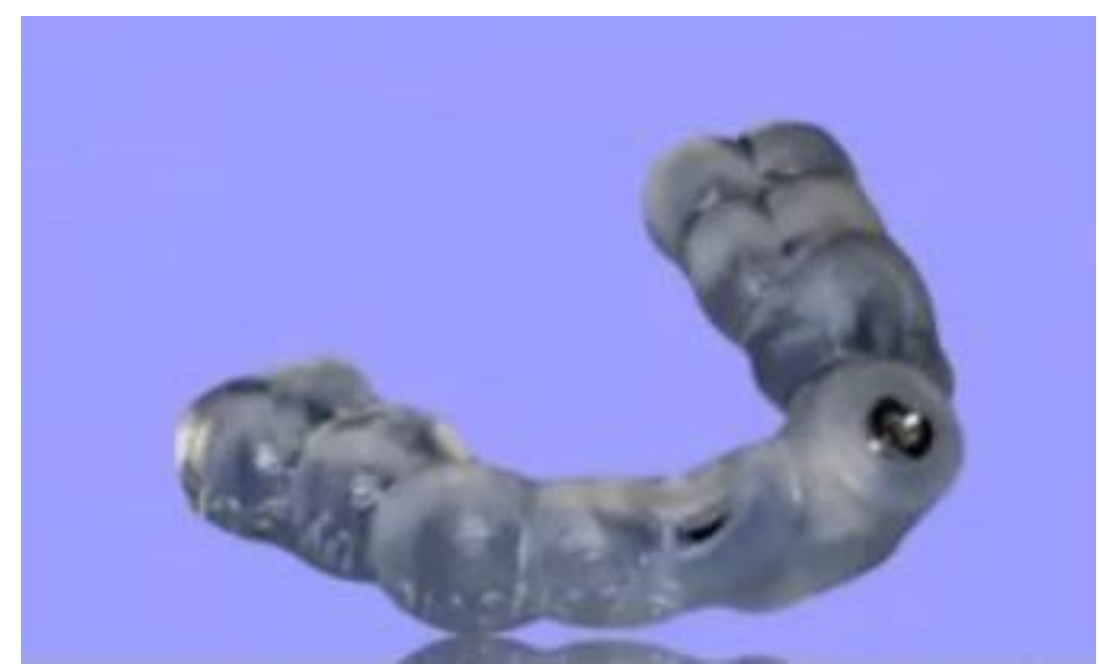

Fonte: KRASTL et al. (2016) 
Figura 13: Modelo bem ajustado na posição exata

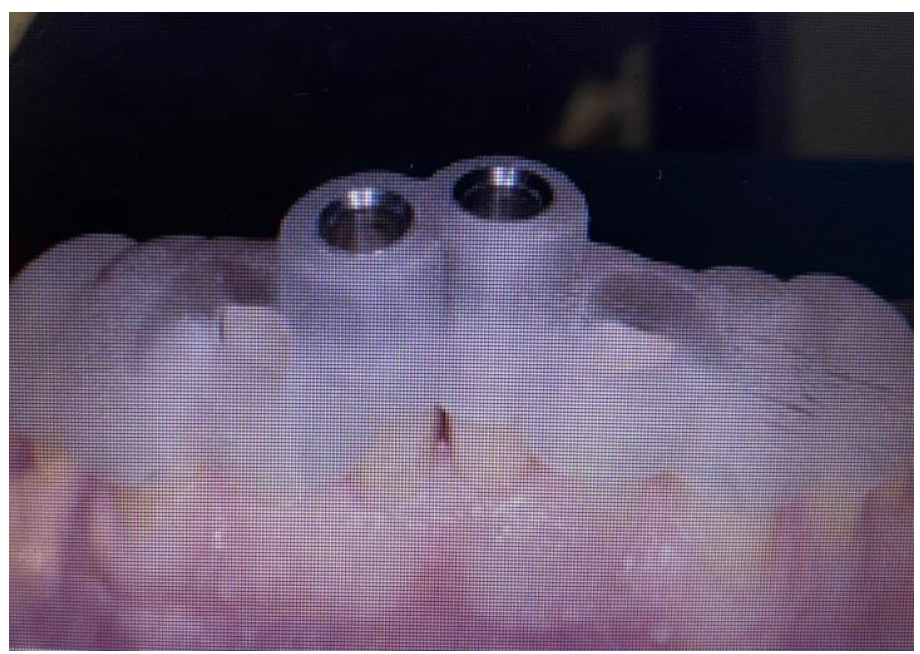

Fonte: Zhender et al. (2015)
Figura 14: Aplicação clínica: Após a remoção do esmalte, a broca foi guiada através da manga para obter acesso ao terço apical do canal radicular. Batente mecânico da manga aponta que a broca atingiu a posição planejada

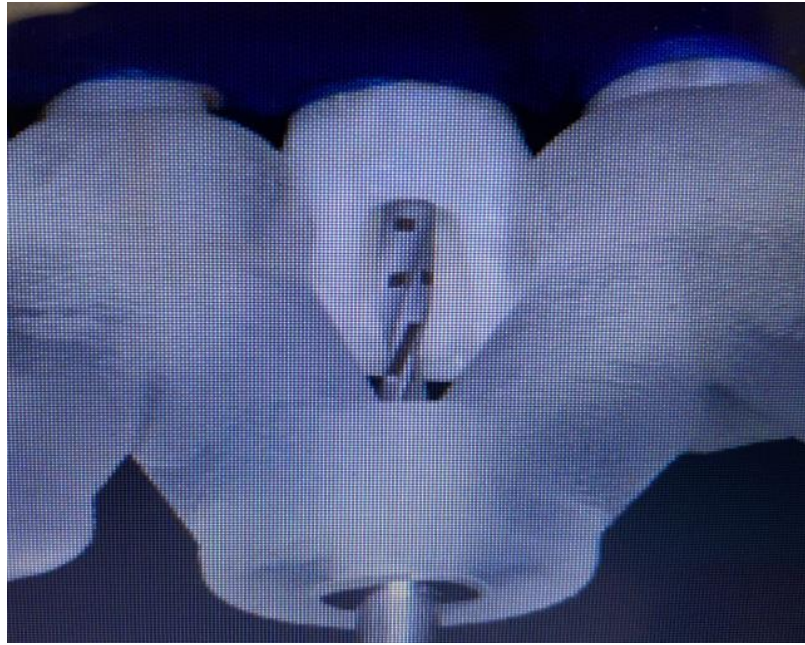

Fonte: Zhender et al. (2015)

Após esse processo, vem à fase do tratamento onde é iniciada na remoção do esmalte com uma broca diamantada até a dentina ser exposta. Após a remoção do esmalte, utilizase a broca microguiada personalizada com diâmetro de $1,3 \mathrm{~mm}$ e comprimento de $20 \mathrm{~mm}$ é usada em uma média de $10.000 \mathrm{rpm}$ com movimentos de bombeamento para obter acesso ao canal radicular, guiada pelo modelo impresso, foi feito com peça de mão de baixa rotação a $10.000 \mathrm{rpm}$. O eixo da broca é inclinado de maneira que a ponta da broca estendida alcance o ápice radiograficamente visível do dente 12. (ZEHNDER M. 2015)

Radiografias convencionais são feitas para garantir que a angulação da preparação está correta. Conforme o plano virtual, a cavidade de acesso, é finalizada quando a broca chega à manga, caracterizando o guia endodôntico (figura 5). (CONNERT T. 2017). 
Figura 15: Procedimento clínico para endodontia microguiada: (A) situação préoperatória, (B) adaptação do molde, (C) retirada do esmalte até a dentina exposta, (D) gabarito com luva incorporada, (E) preparação guiada através da luva e (F) cavidade de acesso de acordo com o planejamento

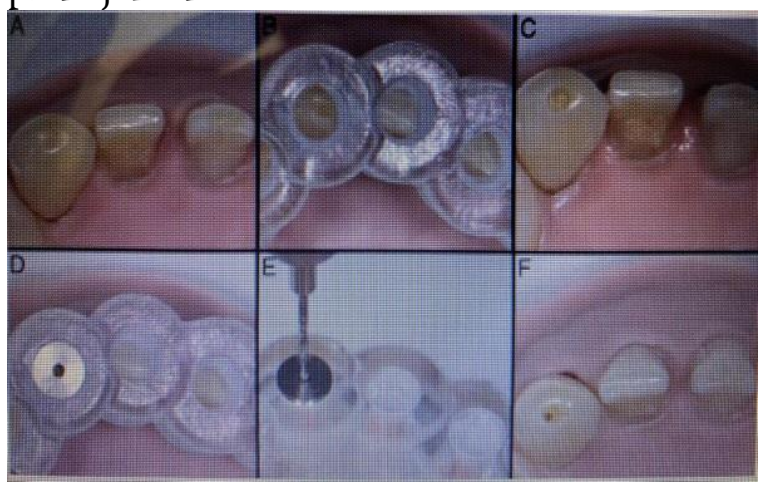

Fonte: CONNERT et al. (2017)
Figura 16: Acesso ao terço apical do elemento dentário, após uso do guia e brocas de haste longa com comprimentos compatíveis com a medição da TCFC, de forma minimamente invasiva

Ao dominar o desafio do acesso, o operador poderá beneficiar-se de limpeza facilitada, modelagem e preenchimento do sistema de canais radiculares, e concluir o tratamento endodôntico normalmente.

Figura 17: A: Modelo posicionado nos dentes superiores para verificar seu ajuste correto e reproduzível; B: Vista da cavidade de acesso endodôntico após o canal radicular localização.

A

B

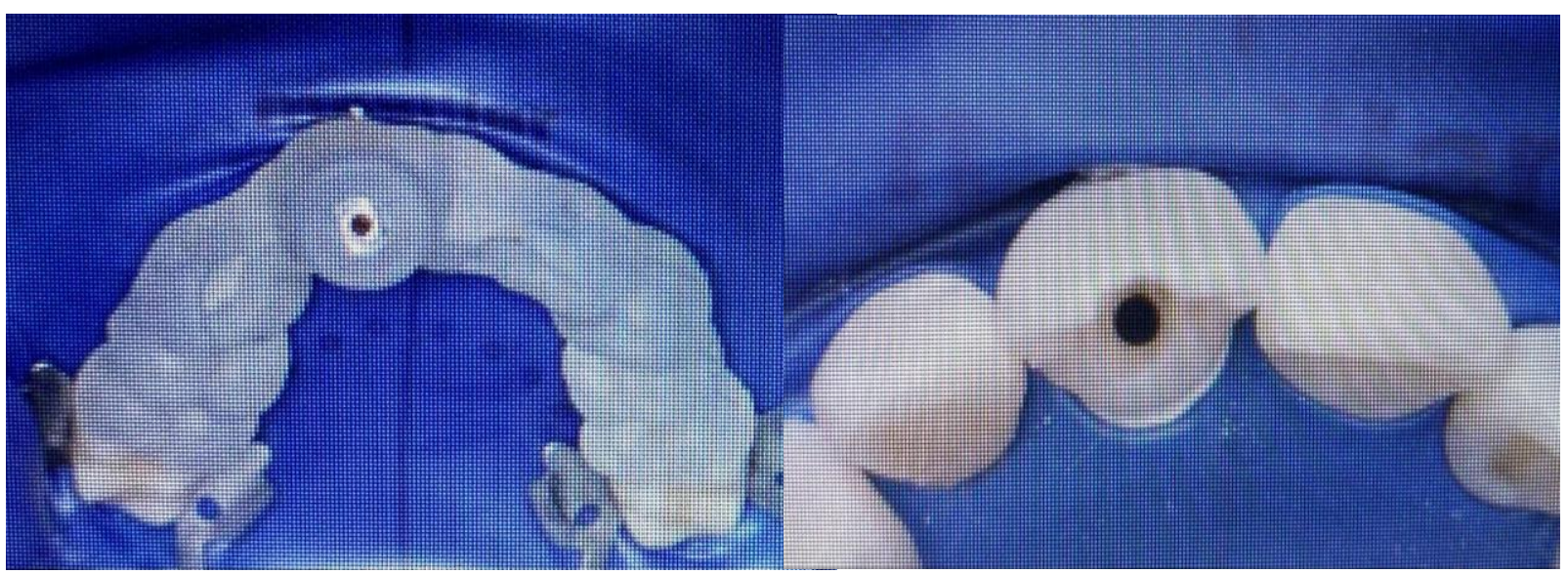

Fonte: KRASTL et al. (2or6 
Figura 18: A: Radiografia de controle com cone de prata no canal radicular; B: Exame radiográfico pós-operatório.

A

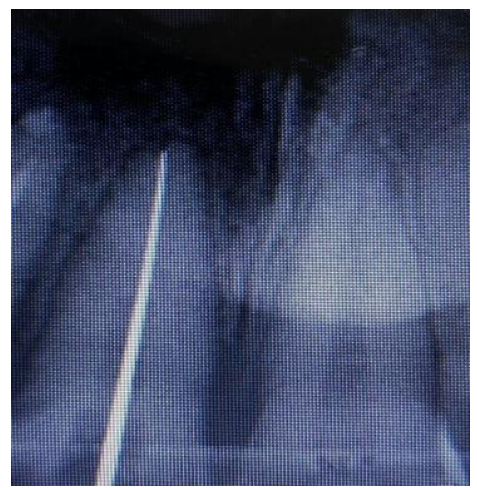

B

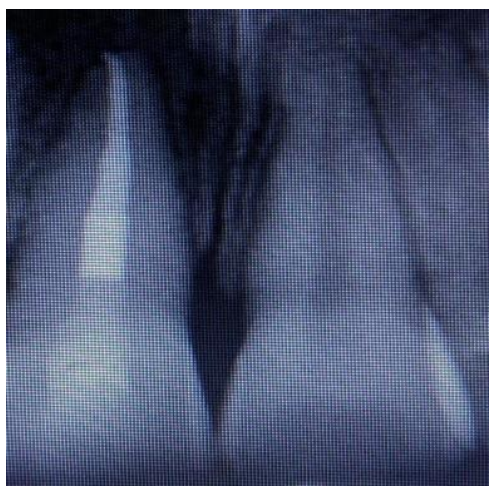

Fonte: KRASTL et al. (2016).

É avaliada como de muita simplicidade, com redução do nível de dificuldade por parte do operador, podendo, consequentemente, ser realizada até por profissionais menos experientes, visto que dispensa uso de um microscópio operatório e técnica elaborada, possibilitando maior preservação da estrutura dental. (MENDES L. 2018)

As vantagens do uso de uma endodontia guiada são que ela reduz os riscos de perfuração da raiz e desvios do canal, fratura de instrumentos e fratura da raiz. Concede um acesso conservador planejado e guiado, ajustado com o mínimo conceito invasivo, limitando perda maciça de estrutura dentária, que está relacionada a um maior risco de fratura. (TAVARES W. 2018)

Também é vista uma técnica custo-efetiva, principalmente quando se comparam aos custos necessários para o planejamento tridimensional e fabricação do modelo, com custos adicionais necessários caso a terapia convencional venha a fracassar, levando à perda dentária. (KRASTL G. 2016)

\subsection{Desvantagem na Endodontia Guiada}

Algumas desvantagens foram observadas nos estudos, o tempo gasto para todo o processo de planejamento virtual, desde a tomografia até a impressão do modelo é discutido por diversos autores quando confrontado ao tratamento convencional dos canais, 
no entanto, o acesso dos canais calcificados com ou sem microscópio operatório, consumiu o mesmo tempo ou até mais em relação a técnica do guia endodôntico, porém mais estudos são necessários em relação a esta hipótese.(CONNERT T. 2017)

Nesta técnica encontra-se algumas limitações como os dentes que possuem restaurações de coroa total o que pode diminuir a precisão das imagens intraorais ${ }_{3} \mathrm{D}$ devido a sobreposição de imagens. Outro desafio no uso dos guias é obtenção da irrigação ideal durante procedimento de osteotomia uma vez em que há espaço para introdução do instrumento utilizado para irrigação. (ACKERMAN S. 2019)

Um fator importante a ser considerado é a elevada dose de radiação na qual o paciente é exposto devido a TCFC. Por isso essa tecnologia deve ser uma alternativa para circunstâncias específicas como calcificação ou morfologia atípica identificada em radiografia dentária. Novos dispositivos de TCFC com um campo de visão limitado apresenta uma dose de radiação mais baixa, porém essa ainda permanece alta em comparação as radiografias convencionais. Todavia frente a um tratamento Endodôntico de maior complexidade, é necessário um número elevado de tomadas radiografias para determinar a localização do canal radicular, ou mesmo tratamento subsequente para controlar a perda dentária anterior, na adolescência pode levar a uma dose geral de radiação maior. (CONNERT T. 2017), (KRASTL G. 2015)

Outra desvantagem potencial do uso da broca para localização de canais radiculares é o risco de fissuras e rachaduras dentárias. Para evitar essa iatrogenia é necessário um cuidado de irrigação abundantemente a broca, a fim de evitar desgastes desnecessários e microfissuras durante a perfuração da dentina, os movimentos de vai e vem, associados avanços graduais, ajudam a prevenir cargas excessivas nas paredes dentários. Ainda em relação às brocas empregadas na endodontia guiada está descrito a necessidade em se desenvolver brocas mais adequadas para o uso em Endodontia Visto que as brocas utilizadas são destinadas a implantodontia. Entretanto apesar das brocas empregadas não serem as ideais para endodontia estas provocaram o menor desgaste da estrutura dentária se comparado com a técnica de se realizar o acesso de forma convencional. (KRASTL G. 2015), (TAVARES W. 2018), (MENDESS L. 2018) 
Para que a confecção da cavidade de acesso seja feita em linha reta paralela ao longo eixo do dente há relatos na literatura a respeito de necessidade da remoção da borda incisal do dente ${ }^{31}$. Para que tal evento seja evitado propuseram uma modificação no modelo para permitir a preparação do acesso padrão para dentes anteriores superiores iniciando o acesso de modo convencional pela palatina ${ }^{29}$. Em outro estudo demonstraram que o acesso Endodôntico guiado em dentes anteriores pode ser alterado com sucesso para evitar dano incisal, alterando a direção da broca durante o planejamento virtual ${ }^{28}$. (CONNERT T. 2018), (TAVARES W. 2018), (MENDESS L. 2018)

Sendo assim, uma limitação dessa tecnologia é que a perda de substâncias e modificação da geometria natural do canal radicular de acordo com a dimensão da troca devem ser aceitas, sendo obrigatório para permitir um preparo guiado e um acesso em linha reta em canal radicular. Porém esta técnica só é praticável em raízes retas ou na parte reta de uma raiz curva. (KRASTL G.2015)

A pesquisa envolvendo Endoguide deve se concentrar no aperfeiçoamento do modelo de guia, incluindo marcadores para a profundidade da cavidade de acesso, cálculo automático na direção de perfuração ideal remoção eficaz de anilhas de dentina, estabelecer acesso em linha reta dadas as condições do canal radicular coronal. (CHEN H. 2018).

Por fim verificou-se que a literatura converge quanto a sua eficácia praticidade e segurança e aplicabilidade dessa técnica. Porém, faz necessário, o desenvolvimento de materiais específicos para Endodontia em especial brocas miniaturizadas, como também estudos retrospectivos relacionados ao sucesso da terapia em longo prazo. (KRASTL G.2015)

\section{CONCLUSÃO}

A Endodontia guiada se apresenta como uma alternativa eficaz e necessária para solucionar casos mais complexos, como tratamento de canais radiculares calcificados, microcirurgias endodônticas, no tratamento de canais calcificados ela é auxiliada pela TCFC, de forma conservadora, reduzindo riscos, permitindo o tratamento do canal radicular, tal como preservando-o na cavidade bucal. A abordagem da endodontia guiada 
aparenta ser um método seguro e clinicamente viável para localizar canais e evitar perfuração radicular em dentes com calcificação pulpar.

Essa técnica ainda apresenta algumas limitações, como a necessidade da fabricação de brocas especialmente adaptadas para a endodontia, sendo que as utilizadas na atualidade são brocas para implantes, entretanto estudos afirmam que essas são evidencialmente eficaz e seguras. Na literatura há relatos de propostas para a modificação no protocolo do tratamento endodôntico guiado, o qual auxiliam a contornar esta problemática. Ademais, ainda que o endoguide possua algumas negações devido ao seu custo elevado, maior tempo de planejamento, exposição a maior nível de radicação devido a TCFC, o uso dessa técnica garante um melhor prognóstico para o dente tratado, logo para o paciente, o que evitaria a necessidade de tratamentos complexos futuro, sendo, portanto, uma técnica com melhor custo-benefício.

\section{REFERÊNCIAS}

AMERICAN ASSOCIATION OF ENDODONTISTS. Aae endodontic case difficulty assessment and referral 2005. Available at: https://www.aae.org/uploadedfiles/publications and research/endodontics colleague s_for_excellence newsletter/ssosecfe.pdf. Accessed february 2, 2016.

ABELLA F. endodontic applications of cone beam computed tomography: case series and literature review, giornale italiano di endodonzia, v.29, n.2, p.38-50, 2015.

ACKERMAN S. Accuracy of 3-dimensional- printed endodontic surgical guide: a human cadaver study. J. Endod, v. 45, n. 5, p. 615-618, may 2019.

ALI A. Arslan H. Guided endodontics: a case report of maxillary lateral incisors with multiple dens invaginatus. Restor dent endod, v.44, n. 4, p.38, oct. 
BUCHGREITS J; Buchgreits M; Mortensen D; Bjorndal L. Guided access cavity preparation using cone-beam computed tomography and optical surfasse scans - an ex vivo study int endod j. 2016.

CAGLAYAN F; Dagistan S; Keles M. the osseous and dental changes of patients with chronic renal failure by cbct. Dento maxillo fac radiol; v.44: 20140398, 2015.

CAMPOS M. Canais calcificados: abordagem em endodontia. Universidade fernando pessoa, porto, v. I, n. I, p. I-43, 2016.

CARROTTE P. Surgical endodontics. British dental journal,198(2), pp. 71-79, 2005.

CASADEI B. Access to original canal trajectory after deviation and perforation with guided endodontic assistance. Aust endod j, v. 46, n. I, p. IоIı6, 2020.

CHEN H. Printing of drill guide template for access cavity preparation um human molars: a preliminary study. Rapid prototyping j, v. 24, n.5, p. 914-919, july. 2018.

COHEN S; Hargreaves K. Cohen caminhos da polpa. ıo edição. Rio de Janeiro, 2orı

CONNERT T. Al microguided endodontic treatment method to achieve minimally invasive access cavity preparation and root canal location in mandibular incisors using a novel computer-guided technique. International endodontic journal, v.51, p.247-255, 2018.

CONNERT T. Microguided endodontics: accuracy of a miniaturized technique for apically extended access cavity preparation in anterior teeth. Journal of endodontics, v. 43, pág. $787-90,2017$

DORANALA S. Endodontic management of canal calcification in maxillary central incisor using $3 \mathrm{~d}$ prototyping technique: a case report. Journal of advanced oral research, $v$. II, n. I, p. 232020682090165I, may 2020. 
KRASTL G. Endodontics: a novel treatment approach for teeth with pulp canal calcification and apical pathology - case report, dental traumatology, v.32, p.240-246, 2016.

KRASTL G. Guided endodontics: a novel treatment aproach for theeth with pulp canal calcification and apical pathology. Dent traumatol,v 32, n.3, p. 240-246, out.2015.

LOPES H; Siqueira J. Endodontia: biologia e técnica. 4ª ${ }^{-}$Edição. Rio de janeiro, 2015.

LUUKKO, K.; KETTUNEN, P.; FRISTAD, I.; BERGGREEN, E. Estrutura e Funções do Complexo Dentino-Pulpar. In: COHEN. Caminhos da Polpa. ıo ${ }^{\underline{a}}$ ed. Rio de Janeiro: Elsevier, 2011. p. 418-46319.

MCCABE P; Dummer P. Pulp Canal Obliteration: An Endodontic Diagnosis And Treatment Challenge. International Endodontic Journal, V.45, P.177-197, 2012

MEDEIROS, Felipe Bruno Gomes. Calcificações pulpares - características clínicas, imagenológicas e morfológicas: revisão sistemática. 2017. Monografia (Graduação) Departamento de Odontologia, Universidade Federal do Rio Grande do Norte, Natal, 2017.

MENDES L. A new approach for minimally invasive access to severely calcified anterior teeth using the guided endodontics technique, joe, v.44, n.Io, oct. 2018.

MOLERI A; Moreira L; Rabello D. O complexo dentino-pulpar. In siqueira, jr. J.f; lopes, h.p. Endodontia: biologia e técnica. 3. Ed. Rio de Janeiro, 2010.

MOVAHHEDIAN M; Haghnegahdar A; Owii F. How the prevalence of pulp stone in a population predicts the risk for kidney stone. Iranian endodontic journal, v.13, n.2, p.246$250,2018$. 
NEVILLE B; Allen C. Patologia: oral \& maxilofacial-pág 122-I25. Rio de Janeiro, 2004.

OGINNI A. Evaluation of radiographs, clinical signs and symptoms associated with pulp canal obliteration: an aid to treatment. Dental traumatology, 25, pp. 620-625, 2009.

RAVANSHAD S. The prevalence of pulp stones in adult patients of shiraz dental school, a radiographic assessment. Journal of dentistry, v. 16, n. 4, p. 356, 2015.

SATHEESHKUMAR P. Idiopathic dental pulp calcifications in a tertiary care setting in south india. Journal of conservative dentistry: jcd, v.16, 2013.

SCARFE W; Farman A; Sukovic P. Clinical applications of cone-beam computed tomography in dental practice. J can dent assoc, ottawa, v. 72, no.I, p. 75-8o, feb. 2006.

SENER S; Cobankara F; Akgünlü F. Calcifications of the pulp chamber: prevalence and implicated factors, clin oral invest, v.13, p.209-215, 2009.

SOUZA S. Association of sickle cell haemoglobinopathies with dental and jaw bone abnormalities. Oral diseases, v.24, p.393-403, 2018.

TASSOKER M; Magat G; Sener S. A comparative study of cone-beam computed tomography and digital panoramic radiography for detecting pulp stones, imaging science in dentistry; v.48, n.3, p.20I-I2, 2018.

TORRES A. Microguided endodontics: a case report of a maxillary lateral incisor with pulp canal obliteration and apical periodontitis, international journal of endodontics, v.52, p.540-549, 2019. 
ZEHNDER M. Endodontics: accuracy of a novel method for guided access cavity preparation and root canal location. International endodontic journal, p. 1-7, 2015. 